\title{
The Engineering, Production and Quality Assurance of the Inner Barrel Staves for the Upgrade of the ALICE Inner Tracking System
}

\author{
Antoine JUNIQUE ${ }^{1}$ on behalf of the ALICE Collaboration \\ CERN \\ CH-1211 Geneva, Switzerland \\ E-mail: antoine.junique@cern.ch
}

\begin{abstract}
A major upgrade of the ALICE Detector is underway during LHC Long Shutdown 2 (2019-2020). This includes a new Inner Tracking System (ITS) consisting of seven cylindrical layers of CMOS Monolithic Active Pixel Sensors. The building blocks of each layer are azimuthal elements called Staves. The Inner Layer Staves are made of a carbon fiber support structure (spaceframe), a carbon fiber cold plate and a Hybrid Integrated Circuit (HIC) consisting of Pixel Chips and passive components bonded onto an aluminium polyimide Flexible Printed Circuit (FPC).

This contribution describes the Inner Layers Staves, the manufacturing processes, the quality assurance methodologies and some lessons learned.
\end{abstract}

Topical Workshop on Electronics for Particle Physics (TWEPP2019)

02 - 06 Septembre 2019

Santiago de Compostela, Spain

\footnotetext{
${ }^{1}$ Speaker
} 


\section{Introduction}

A major upgrade of the ALICE Detector is underway during LHC LS2 (2019-2020) to enhance its measurement and data recording capabilities. This includes a new Inner Tracking System (ITS) [1] which consists of seven concentric cylindrical layers of CMOS Monolithic Active Pixel Sensors [2]. The basic building blocks of the detector are azimuthal segments called Staves, extending over the whole length of each layer. Those of the middle and outer layers are $92 \mathrm{~cm}$ and $154 \mathrm{~cm}$ long, respectively. The radial distances from the beam line of the three innermost layers are 23,31 and $39 \mathrm{~mm}$, respectively. The radial distance of the outermost layer is $39 \mathrm{~cm}$. The staves constituting the three innermost layers (Inner Barrel) are $30 \mathrm{~cm}$ long and identical. They feature an unprecedentedly low material budget of $0.35 \% \mathrm{X}_{0}$. The engineering and manufacturing of the Inner Barrel Staves, and the quality assurance methods are discribed in the following together with challenges, findings and lessons learnt.

\section{Inner Barrel (IB) stave}

The term stave is used to refer to complete detector elements arranged in cylindrical layers. The Inner Barrel (IB) stave consists of the following elements (fig. 1):

- Space frame: Truss-like lightweight mechanical support structure for the single stave based on composite material (carbon fiber).

- Cold Plate: carbon ply that embeds pipes for circulating cooling water.

- Hybrid Integrated Circuits (HIC): assembly consisting of a aluminium polyimide Flexible Printed Circuit (FPC) on which the pixel chips (9 for IB) and passive components are bonded.

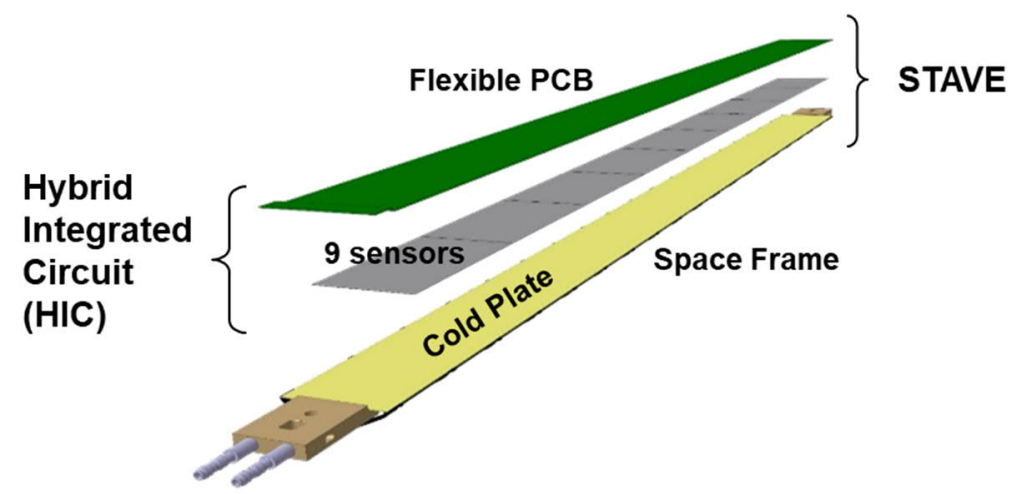

Figure 1: Schematic view of the IB stave.

\section{Inner Barrel Flex Printed Circuit (FPC)}

The FPC is a double side printed circuit with $75 \mu \mathrm{m}$ thick polyimide as substrate. Figures 2 and 3 show the layer stackup. There are two conducting layers of aluminium, $25 \mu \mathrm{m}$ thick. The two cover layers are $20 \mu \mathrm{m}$ thick. This aluminium FPC provides analogue and digital power, a bias voltage, 9 differential lines carrying signals at $1.2 \mathrm{~Gb} / \mathrm{s}$ and 2 differential buses for slow control and clock at $40 \mathrm{MHz}$. The design of the bus layout, which has to satisfy very tight physical and geometrical constraints, required a high level of optimization and simulation. Notably, the highspeed differential lines are routed only on one side, without vias, to optimise the signal integrity. 


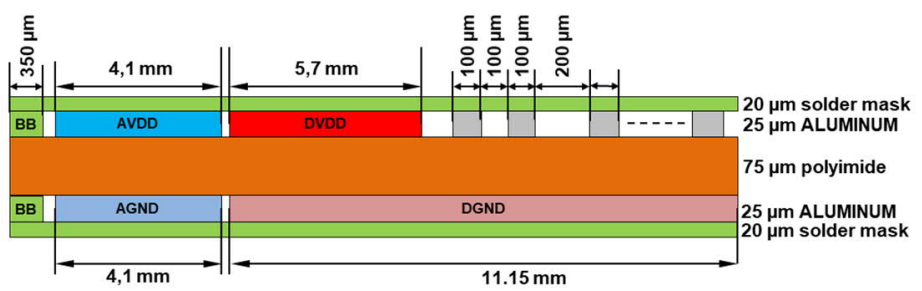

Figure 2: ITS FPC stackup.

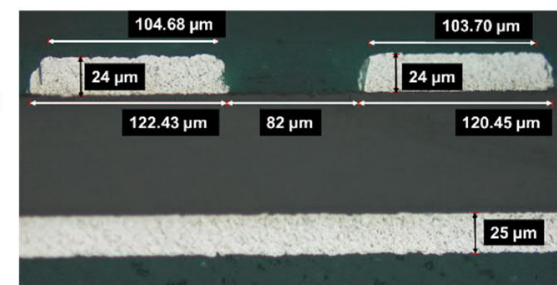

Figure 3: FPC metallographic crosssection.

The FPCs are for the most part manufactured at the CERN PCB workshop, which has developed the use of aluminum instead of the industrial standard copper. The manufacturing process requires 12 main steps with an intensive quality assurance control. The main phases are the following: (a) Stabilization of polyimide by heat treatment in vacuum at $450{ }^{\circ} \mathrm{C}$ for $2 \mathrm{~h},(\mathrm{~b})$ hole drilling by laser, (c) FPC metrology, (d) preparation for aluminium coating polishing and cleaning, (e) aluminium PVD (Physical Vapour Deposition) coating, (f) FPC metrology, (g) photo-lithography, (h) chemical etching, (i) coverlay and $\mathrm{Ni} / \mathrm{Au},(\mathrm{j})$ outline cutting by laser, (k) electrical tests and metallographic analysis, (l) manual soldering of passive components, (m) FPC metrology, (n) final cleaning and visual inspection.

\section{Inner Barrel Hybrid Integrated Circuit Assembly}

The HIC assembly is achieved with the help of a custom made assembly and test machine. This machine, named ALICIA, was designed and produced by an external company following CERN specifications. It is equipped with a probe card to electrically test each pixel chip. A robotic arm allows the automated picking, testing and positioning of the chips on jigs. There are 9 chips, $50 \mu \mathrm{m}$ thick, in the case of the Inner Barrel modules. There are 14 chips, $100 \mu \mathrm{m}$ thick, in the case of the modules used for the Outer Barrel. An automated visual inspection is also done to verify the chip quality and cleanliness. The HIC assembly sequence is the following: (a) chip alignment (5 $\mu \mathrm{m}$ accuracy) and edge inspection in ALICIA, (b) preparation of the glue in a planetary mixer 30 min before dispensing, (c) glue distribution on the FPC using a $90 \mu \mathrm{m}$ thick gluing mask (fig. 4), (d) placing the FPC over chips on the jigs using $50 \mu \mathrm{m}$ shimming, (e) glue curing for 12 hours, (f) removal from ALICIA and visual inspection (fig. 5). To reduce the dead area, the pixel chips are placed with an edge-to-edge distance of only $100 \mu \mathrm{m}$. The edge of the chip is at $10 \mu \mathrm{m}$ only from the active seal-ring.

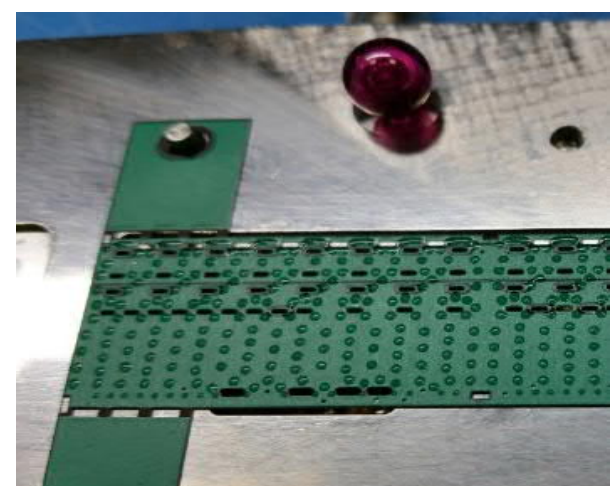

Figure 4: Droplets of glue after distribution over the FPC.

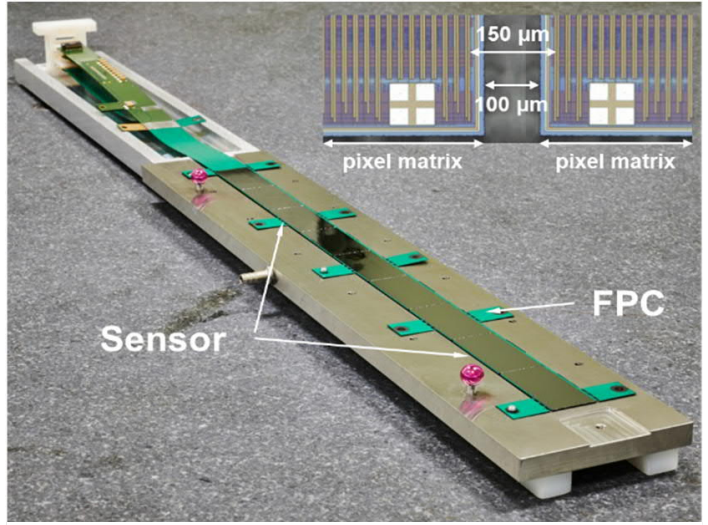

Figure 5: HIC back side after chips gluing. 
The Enginneering, Production and QA of the Inner Barrel Staves for the Upgrade of the ALICE Inner Tracking System

\section{Electrical connections}

The connection between the FPC and the pixel chips are made via ultrasonic wire bonding. Power supply connections are distributed over the full surface of the chip while the I/O pads are concentrated along one edge of the chip. Because of the specific topology of the pads and the presence of only two metal layers, bonding wires must pass through holes in the FPC itself as shown in figures 6 and 7.

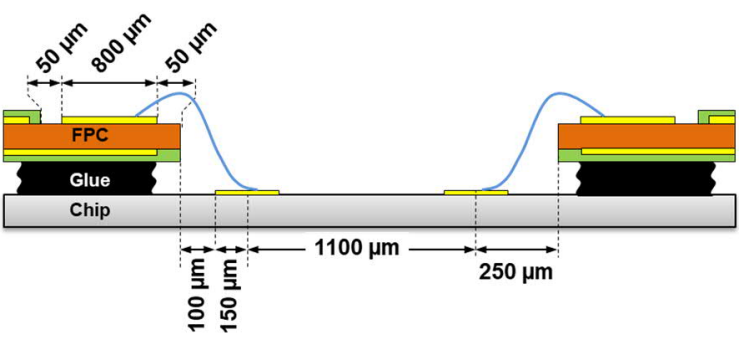

Figure 6: Schematic view of the wire bonding.

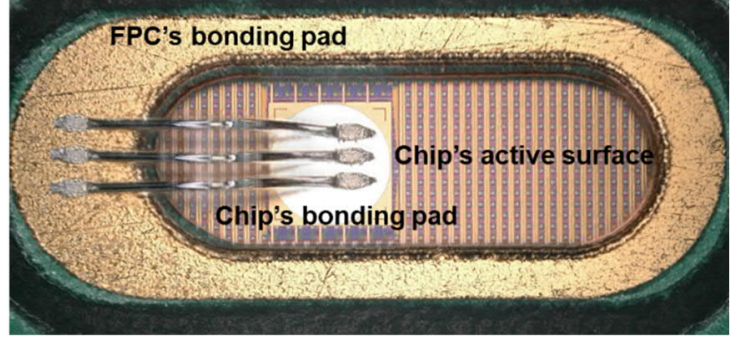

Figure 7: Photography of the wire bonding through via.

After the electrical connections are done, the HIC is intensively tested. Firstly, the power supply is connected and the supply voltage is slowly increased to its nominal value. At the same time, the backside of the chip is inspected with a thermal camera to detect potential short-circuits. Secondly, a complete readout test is carried out in order to qualify functionally the HIC.

\section{Inner Barrel Stave assembly}

The stave (fig. 8) assembly follows the same principles of the HIC assembly. Glue is distributed over the cold plate surface and the HIC is placed over it. The assembly sequence is the following: (a) glue preparation in a planetary mixer 30 min before applying, (b) distribution of the glue and gluing of the HIC over the cold plate, (c) visual inspection, (d) power test and readout test, (e) stave metrology.

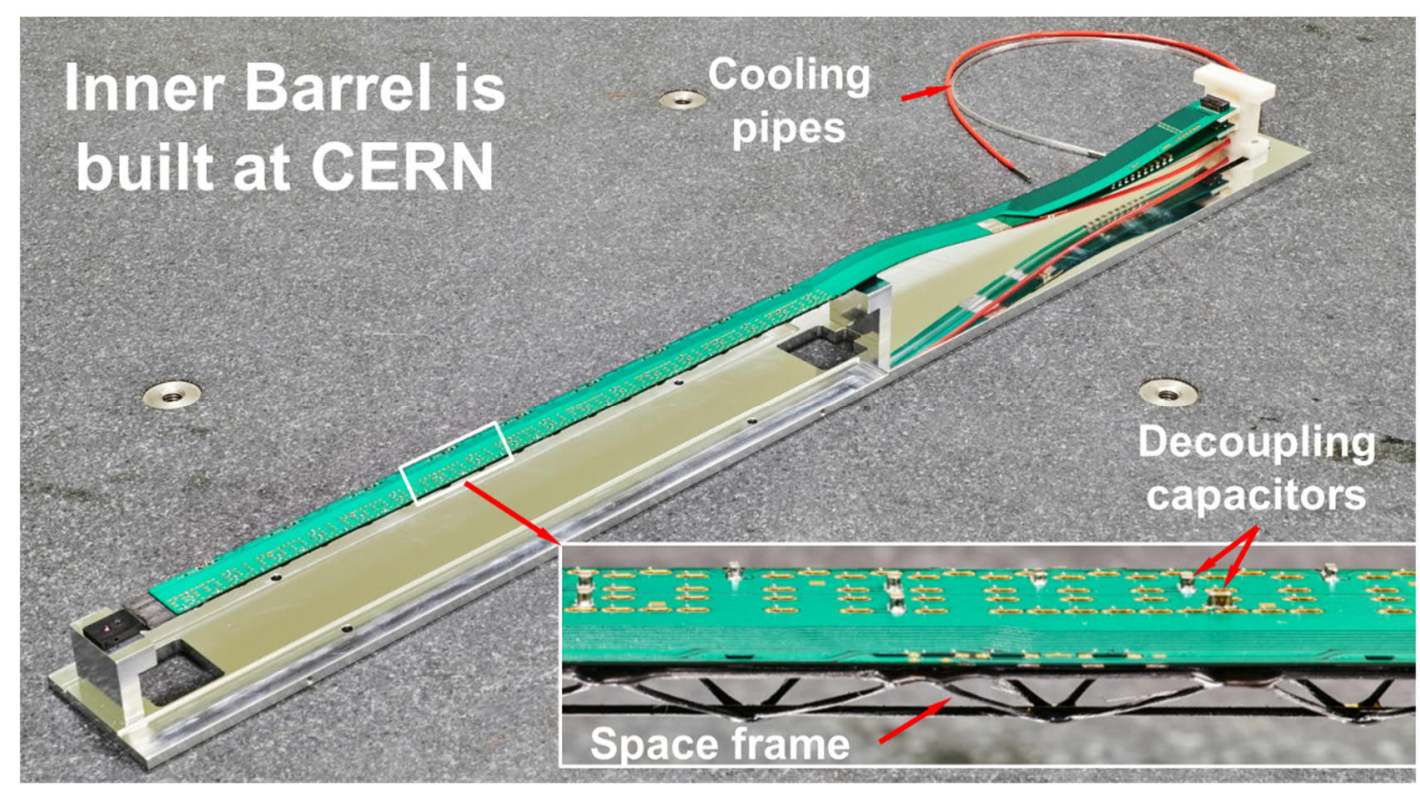

Figure 8:Photography of an IB Stave on its support structure and ready for installation. 


\section{Challenges}

The construction of the ALICE ITS IB staves has required intensive monitoring at each stage of their production to ensure the required mechanical, thermal and electrical quality and their longterm stability. The layout and manufacturing method of the gluing mask required thorough optimization to prevent glue seepage over the bond pads and eliminate contaminants (fig. 9). In order to meet the tight dimensional requirements on the chips alignment with respect to the FPC (fig. 10), the layout of the FPC had to take in account the dimensional variations of the FPC due to the FPC manufacturing process. Additionally, the FPC substrate had to be thermally stabilized prior to processing and the manufacturing steps with thermal stress minimized. Sub-millimetric particles of contaminants (Fig. 11) were found to cause mechanical and electrical damages to the chips. Intensive monitoring and tracing of the sources of contamination were carried out to minimize role of these events.

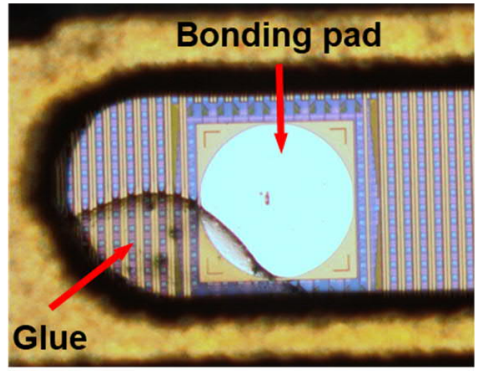

Figure 9: Glue seepage cover the bonding pad.

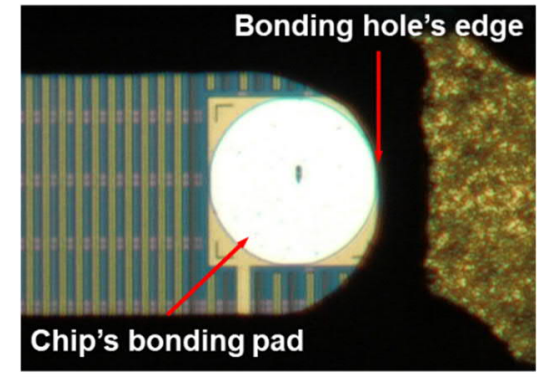

Figure 10: Chip's bonding pad misalignment with respect to FPC.

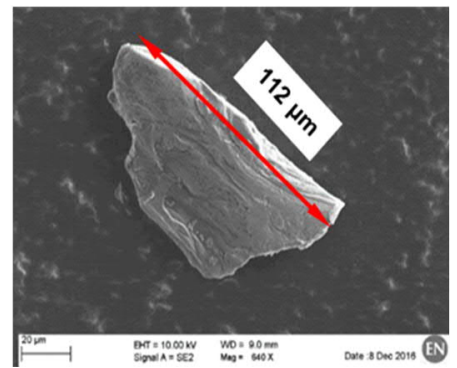

Figure 11: Aluminium particle coming from gluing mask manufacturing process.

\section{Conclusion}

The manufacturing of $2 \times 48$ staves for the new ALICE ITS Inner Barrel required thorough developments and intense quality assurance during the production. The IB staves feature a radiation length of $0.35 \% \mathrm{X}_{0}$. A combination of unconventional materials and technics was employed, including two-sided aluminium based FPC, bonding through holes and gluing over the active side of the CMOS sensors. To achieve this result, intense quality assurance at every phase was mandatory. Each manufacturing step had to be carefully monitored with appropriate technics including metrology, electrical testing, visual inspection, cross-section, SEM analysis and metallography analysis. The commissioning of the new ALICE ITS has begun in mid 2019. It will be completed by April 2020 and followed by the installation of the detector in the experimental cavern in summer 2020.

\section{References}

[1] ALICE collaboration, Technical design Report for the Upgrade of the ALICE Inner tracking system, J. Phys. G, 41 (2014), Article 087002.

[2] M. Suljic, Journal of Instrumentation, Volume 11, November 2016, 2016 JINST 11 C11025, ALPIDE: the Monolithic Active Pixel Sensor for the ALICE ITS upgrade 